\title{
Ruptured cerebral pseudoaneurysm in an adolescent as an early onset of COVID-19 infection: case report
}

\author{
Dragan Savić ${ }^{1}$ (D) $\cdot$ Tarik M. Alsheikh $^{1} \cdot$ Ahmad Kh. Alhaj $^{1} \cdot$ Lazar Lazovic $^{2} \cdot$ Lamya Alsarraf $^{2} \cdot$ Petar Bosnjakovic $^{2}$. \\ Waleed Yousef ${ }^{1}$
}

Received: 15 July 2020 / Accepted: 20 July 2020 / Published online: 27 July 2020

(C) Springer-Verlag GmbH Austria, part of Springer Nature 2020

\begin{abstract}
The clinical manifestations of coronavirus disease 2019 (COVID-19) are non-specific and multi-inflammatory. They vary from mild to severe manifestations that can be life-threatening. The association of SARS-CoV-2 infection and pseudoaneurysm formation or rupture of an already existing aneurysm is still unexplored. Several mechanisms may be involved, including the direct destruction to the artery by the viral infection or through the release of the inflammatory cytokines. We are presenting a case of a 13-year-old girl with a ruptured cerebral pseudoaneurysm of the left middle cerebral artery (M2 segment) with severe intracerebral hemorrhage as the earliest manifestation of COVID-19 infection.
\end{abstract}

Keywords Cerebral Pseudoaneurysm · COVID-19 infection · Adolescent

$\begin{array}{ll}\begin{array}{l}\text { Abbreviations } \\ \text { SARS-CoV-2 }\end{array} & \begin{array}{l}\text { Severe acute respiratory syndrome } \\ \text { coronavirus 2 }\end{array} \\ \text { MCA } & \text { Middle cerebral artery } \\ \text { GCS } & \text { Glasgow coma score } \\ \text { CT } & \text { Computed tomography } \\ \text { CTA } & \text { Computed tomography angiography } \\ \text { DSA } & \text { Digital subtraction angiography } \\ \text { CXR } & \text { Chest X-ray }\end{array}$

\section{Introduction}

The coronavirus disease 2019 (COVID-19) pandemic is a viral infection that mainly affects the respiratory tract [24]. It can also cause multiple inflammatory reactions with a wide range of clinical manifestations in different organs [23]. The vascular

This article is part of the Topical Collection on Vascular Neurosurgery Other

Dragan Savić

dr.dragan.savic@gmail.com

1 Department of Neurosurgery, Ibn Sina Hospital, Ministry of Health, Kuwait City, Kuwait

2 Medical Imaging Department, Ibn Sina Hospital, Ministry of Health, Kuwait City, Kuwait complications after infection with this virus have been reported, but the possible association with cerebrovascular consequences, particularly intracranial hemorrhages, is still under debate [11, 16]. There are rare case reports of adult patients (the youngest was a 31-year-old male, other patients over 60 years old) with COVID-19 infection and ruptured cerebral aneurysm with subarachnoid hemorrhage $[1,16,19]$. The authors assumed that these conditions could be causally linked. However, preceding information from the severe acute respiratory syndrome (SARS) epidemic in 2003 proposed a higher incidence of stroke [3, 14, 21]. Besides, some other viruses are associated with cerebral aneurysmal arteriopathy, such as human immunodeficiency virus (HIV), varicella zoster virus, cytomegalovirus, hepatitis $\mathrm{C}$ virus, and parvovirus B-19 $[4,13]$. It is proposed that one-third of all vasculopathy conditions are caused by infectious vasculitis of medium and large intracranial vessels [13].

In this paper, we present an adolescent girl with COVID-19 infection, who developed an intracerebral hematoma due to cerebral pseudoaneurysm rupture. We speculate that the formation and the rupture of cerebral pseudoaneurysm could be linked to COVID-19 infection.

\section{Case presentation}

A 13-year-old Syrian girl, previously healthy, was admitted to the emergency department after a sudden loss of 
consciousness that was followed by the development of rightside weakness. At the time of admission, the Glasgow coma score (GCS) was 12. She was opening eye spontaneously, obeying commands, and only moaning. The motor power of the right limbs was 0 out of 5 . Urgent non-enhanced computed tomography (CT) of the brain revealed a large left-side frontoparietal intracerebral hematoma with an intraventricular extension and a midline shift of $5 \mathrm{~mm}$ to the contralateral side (Fig. 1). At this point, computed tomography angiography (CTA) of the cerebral arteries was conducted, and it showed a pseudoaneurysm of the frontoparietal branch of the left middle cerebral artery (MCA) in the M2 segment. Laboratory analyses were normal, except elevated leukocytes $(20.6 \times$ $10^{9} / \mathrm{L}$ ), with low lymphocytes (12\%), low hemoglobin level $(10.6 \mathrm{~g} / \mathrm{dL})$, and slightly elevated lactate dehydrogenase (432 U/L).

Shortly after performing the radiological images, her conscious level was deteriorated. She had been directly intubated and sedated. At that moment, she was shifted to the national neurosurgical center in Kuwait. On admission, the clinical assessment showed that her GCS is seven. Particularly, she was localizing to painful stimuli, but without any verbal or eye responses. The girl was moved to cerebral digital subtraction angiography (DSA) room. The angiography (Fig. 2) demonstrated the left MCA trifurcation. Besides, it confirmed the left MCA pseudoaneurysm of the M2 segment, measuring $8 \times$
$7 \mathrm{~mm}$, with a base of $2.3 \mathrm{~mm}$, and a partial thrombosis. DSA also revealed the dissection of the left M2 segment (length of $3 \mathrm{~cm}$ ).

The unusual presentation of the pseudoaneurysm in an adolescent without any previous traumatic history pushed the neurosurgery team to consider vasculitis due to infection as the etiology. With this in mind and the current pandemic, a nasopharyngeal swab of COVID-19 was taken despite that the patient had no infectious symptoms. On the same day of admission, the decision was to emergently proceed with left frontotemporal craniotomy. We have evacuated the hematoma with trapping the pseudoaneurysm and scarifying the carrying artery (Fig. 3). Postoperative CT brain scan revealed the removal of the clot with a small remnant and without any acute ischemia or rebleeding. Additionally, a new CTA showed the exclusion of the aneurysm from the circulation. Moreover, a right frontal external ventricular drain was inserted 1 day after the primary operation.

After surgery, the patient was much improved. Therefore, she was extubated after the improvement. Straight away, we received the positive result of the polymerase chain reaction (PCR) of severe acute respiratory syndrome coronavirus 2 (SARS-CoV-2). As a rule, she was transferred to a specialized COVID-19 hospital. At that hospital, the patient was isolated in the Intensive Care Unit, and she received the appropriate management. The girl was daily followed by a neurosurgeon.
Fig. 1 Different cuts of computed tomography $(\mathrm{CT})$ brain revealed a large left-side frontoparietal intracerebral hematoma with an intraventricular extension and a midline shift of $5 \mathrm{~mm}$ to the contralateral side
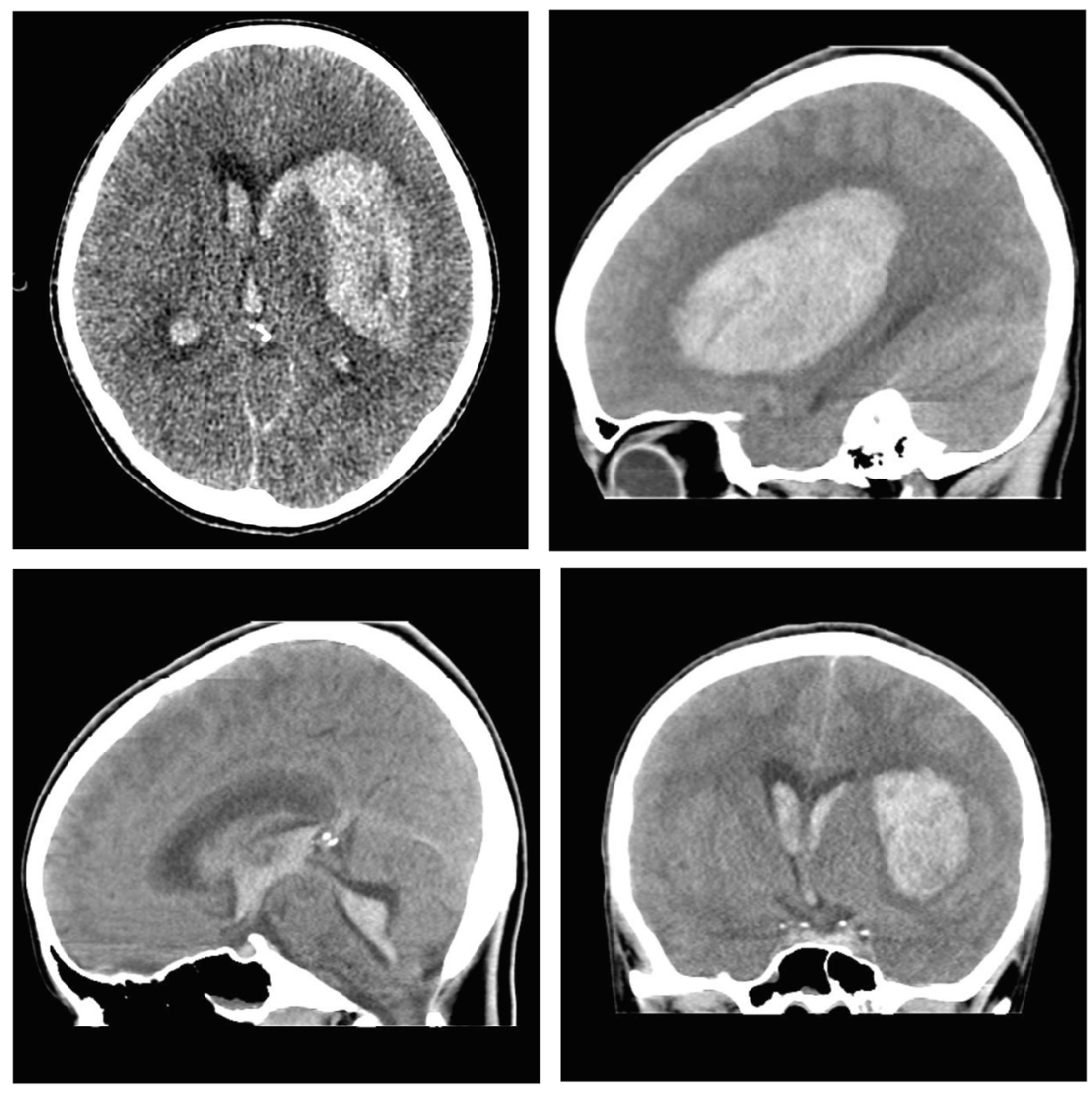
Fig. 2 Cerebral digital subtraction angiography (DSA) images demonstrated the left middle cerebral artery pseudoaneurysm of the M2 segment
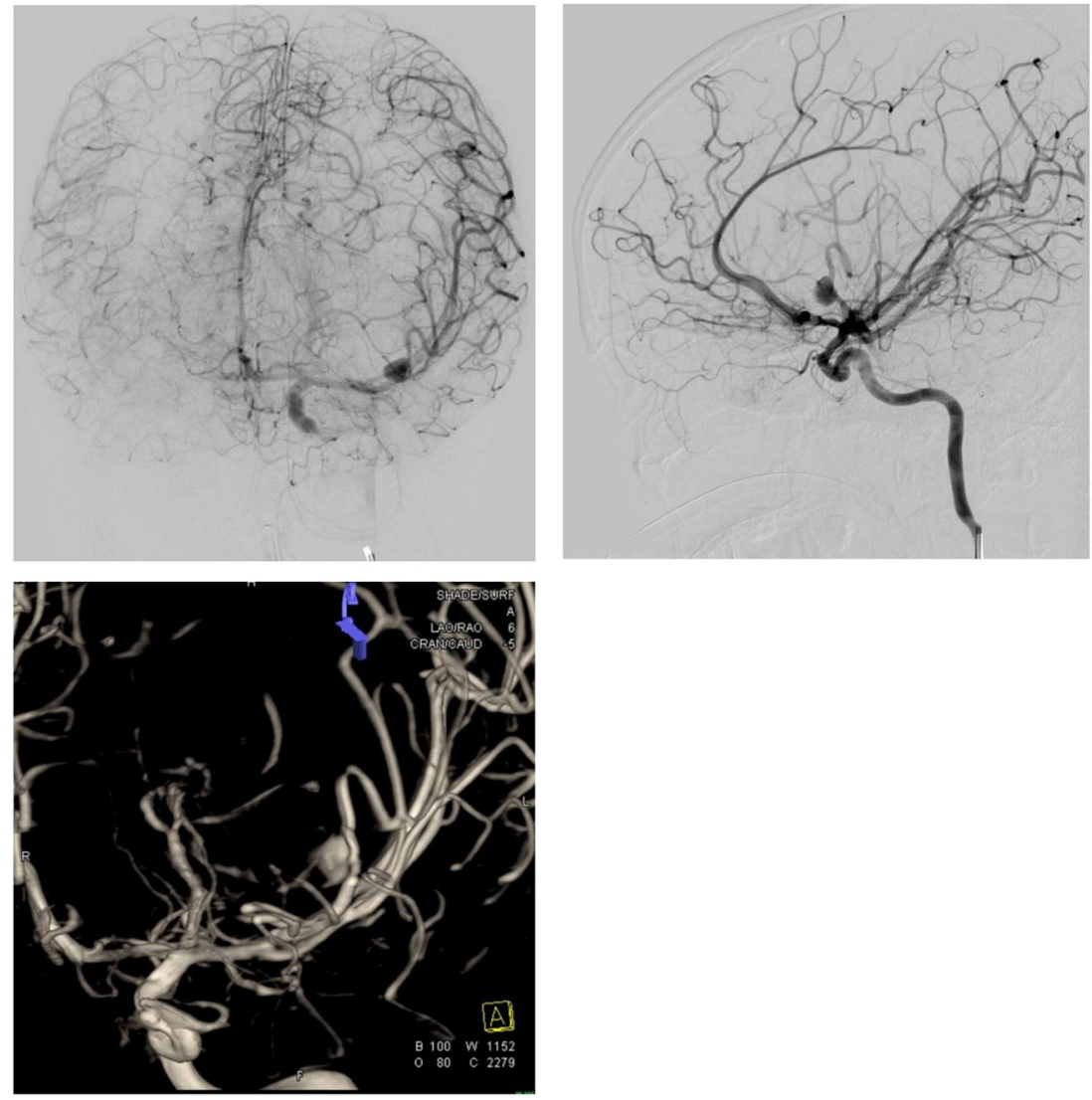

Three days after surgery, the level of consciousness dropped again to GCS of eight so that she was re-intubated. Urgent CT brain on that occasion illustrated the stationary course with slight left-sided brain edema. During the course of hospitalization, she was consistently afebrile. Several follow-up chest $\mathrm{X}$-rays (CXR) showed no pulmonary pathology in the meanwhile.

Ten days after surgery, the patient developed a new onset of high-grade fever. New laboratory results were as follows: WBCs $\left(18.5 \times 10^{9} / \mathrm{L}\right)$ with lymphopenia, C-reactive protein (132) mg/L, LDH (458 U/L), and D-Dimer (2032 ng/ml). A

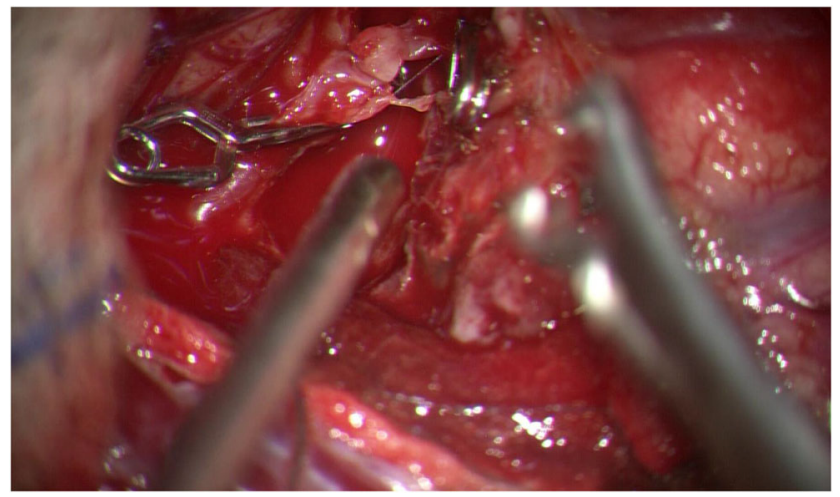

Fig. 3 Intraoperative microscopic image is showing the pseudoaneurysm of the M2 segment of the left middle cerebral artery. Besides, the dissection is shown along the length of the affected artery new CXR showed left apical pulmonary atelectasis. A series of chest radiographs showed progression of the respiratory disease until acute respiratory distress syndrome (ARDS) was confirmed (Fig. 4). On postoperative day 14, the nasopharyngeal PCR test was still positive for SARS-CoV-2. A new follow-up CT brain scan showed hypodensity at the site of hemorrhagic stroke, resorption of blood from lateral ventricles, and decrease of brain edema (Fig. 5). Furthermore, abdominal ultrasound showed hepatomegaly, splenomegaly

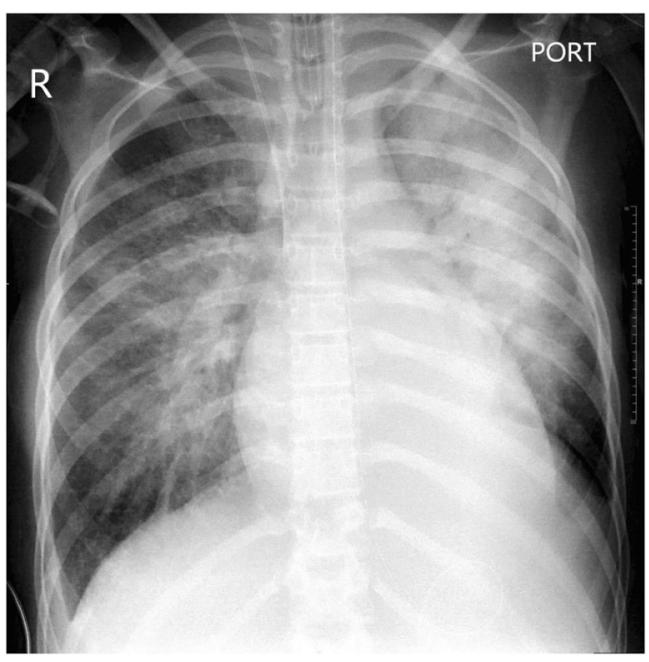

Fig. 4 CXR showed the progression of the respiratory disease 


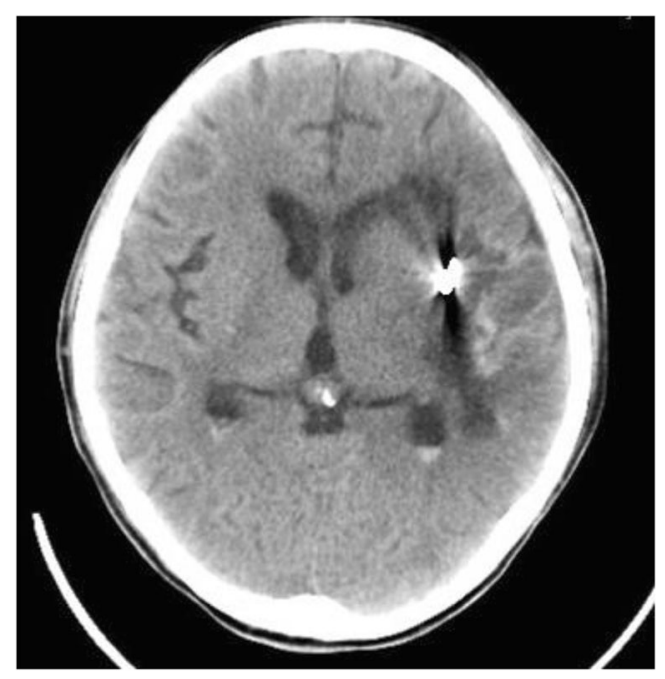

Fig. 5 Postoperative non-enhanced axial CT brain (10 days after the surgery)

with splenic infarction, bilateral nephropathy changes, and ascites. The patient has been in a very critical clinical status due to brain damage as well as ARDS consequences. She was still intubated, on a mechanical ventilator with continuous high-grade fever. Neurological assessment unfolds the very poor prognosis with GCS of four.

\section{Discussion}

As far as we know, this is the first reported case of an adolescent with ruptured cerebral pseudoaneurysm as the initial presentation of COVID-19 infection. There are only rare case reports of a ruptured cerebral aneurysm in adults with this infection; the youngest was 31 years old $[1,16,19]$. Furthermore, a retrospective study of COVID-19 patients with acute cerebrovascular pathologies was published [20]. The total sample size of that study was 22 patients with mean age $59.5 \pm 16.0$ years ( $54.4 \%$ females), among them three cases of aneurysm rupture (majority had an acute ischemic stroke and two patients had cerebral sinus thrombosis). These authors investigated the association of COVID-19 and neurological manifestations (including stroke) and reported that despite timely intervention and favorable reperfusion, the mortality rate in COVID-19 patients with large-vessel occlusion was high [20]. Another condition with meningoencephalitis due to SARS-Cov-2 was complicated by right frontal intracerebral hematoma with subarachnoid hemorrhage [2]. However, the CTA was negative for any vascular malformation or aneurysm in that case [2]. The SARS-CoV-2 virus was not detected by PCR in the cerebrospinal fluid (CSF) of patients with ruptured cerebral aneurysms $[1,16]$. On the other hand, the SARS-CoV genome was identified in the CSF sample of a reported case with SARS in 2003 [12].
The exact pathophysiological mechanisms of the formation of cerebral aneurysms or pseudoaneurysms remain controversial. The intracranial blood vessels are thinner than extracranial. Nevertheless, these vessels have less elasticity in the tunica media and adventitia $[9,22]$. Systemic inflammation is known to cause arterial injury including the breakdown of collagen and permeability of the blood-brain barrier. The inflammatory cytokines may increase the risk of hemorrhagic stroke by weakening the cerebral vessels [15]. The cytokine release syndrome is described during the coronavirus infection [18]. The destruction of the blood vessels could be explained by the direct insult to the artery by the viral infection or through the release of the inflammatory cytokines $[5,15$, 16]. Furthermore, the angiotensin-converting-enzyme-2 (ACE-2) receptors are highly expressed in the endothelial cells of the arteries $[8,10,26]$. The earlier biological researches about this pandemic identified that SARS-COV-2 invades the host cells by attaching to ACE-2 receptors [7]. A recently reported case also supported the association between the novel coronavirus and cerebral vasculitis [11]. The intracranial vessels are vulnerable, and any significant damage can increase the incidence of a brain hemorrhage.

The assumption that some infections can rapidly induce the formation of cerebral pseudoaneurysm or the rupture of an already existing defect is yet unknown. Even though cerebral pseudoaneurysm formation is rare in the absence of trauma, mycotic infections can predispose to pseudoaneurysm [17]. COVID-19 infection was asymptomatic in our patient at the time of presentation. As a consequence of the infection, a multisystem inflammatory syndrome or the direct damage by the virus has resulted in severe brain hemorrhage attributable to the ruptured pseudoaneurysm. The possibility of the rupture of already existing pseudoaneurysm could not be excluded. However, the association between viral infection and mycotic pseudoaneurysm should be considered [6]. A systematic review showed that the most common HIV-associated cerebral pseudoaneurysms were found in MCA [4]. Similarly, our patient had the pseudoaneurysm in the left MCA. Hemorrhages because of pseudoaneurysm rupture are typically followed by high-rate morbidity and mortality [25].

\section{Conclusion}

The full impact of COVID-19 infection on the vascular system is still unclear. Cytokine release syndrome has been identified as a COVID-19-related condition. We consider that it is relevant to draw attention to the possibility of a link between this infection and cerebral pseudoaneurysm formation and/or rupture, especially in adolescents. Epidemiological and clinical studies are required to demonstrate that pseudoaneurysm can be developed as an early sign of SARS-CoV-2. 


\section{Compliance with ethical standards}

Conflict of interest The authors declare that they have no conflict of interest.

Ethical consideration An informed consent about the case report was taken from the parents.

\section{References}

1. Al Saiegh F, Ghosh R, Leibold A et al (2020) Status of SARS-CoV2 in cerebrospinal fluid of patients with COVID-19 and stroke. J Neurol Neurosurg Psychiatry:jnnp-2020-323522

2. Al-Olama M, Rashid A, Garozzo D (2020) COVID-19-associated meningoencephalitis complicated with intracranial hemorrhage: a case report. Acta Neurochir 162(7):1495-1499

3. Arabi YM, Harthi A, Hussein J et al (2015) Severe neurologic syndrome associated with Middle East respiratory syndrome corona virus (MERS-CoV). Infection. 43(4):495-501

4. Baeesa SS, Bakhaidar M, Almekhlafi MA, Madani TA (2016) Human immunodeficiency virus-associated cerebral aneurysmal vasculopathy: a systematic review. World Neurosurg 87:220-229

5. Beyrouti R, Adams ME, Benjamin L et al (2020) Characteristics of ischaemic stroke associated with COVID-19 [published online ahead of print, 2020 Apr 30]. J Neurol Neurosurg Psychiatry: jnnp-2020-323586

6. Carod Artal FJ (2016) Clinical management of infectious cerebral vasculitides. Expert Rev Neurother 16(2):205-221

7. Chen Y, Guo Y, Pan Y, Zhao ZJ (2020) Structure analysis of the receptor binding of 2019-nCoV [published online ahead of print, 2020 Feb 17]. Biochem Biophys Res Commun 525(1):135-140

8. Donoghue M, Hsieh F, Baronas E et al (2000) A novel angiotensinconverting enzyme-related carboxypeptidase (ACE2) converts angiotensin I to angiotensin 1-9. Circ Res 87(5):E1-E9

9. Fang H, Wright IS, Millikan CH (1958) In: a comparison of blood vessels of the brain and peripheral blood vessels. Cerebral vascular diseases. Grune and Stratton, New York, pp 17-22

10. Hamming I, Timens W, Bulthuis ML, Lely AT, Navis G, van Goor $\mathrm{H}$ (2004) Tissue distribution of ACE2 protein, the functional receptor for SARS coronavirus. A first step in understanding SARS pathogenesis. J Pathol 203(2):631-637. https://doi.org/10.1002/ path. 1570

11. Hanafi R, Roger PA, Perin B, Kuchcinski G et al (2020) COVID-19 neurologic complication with CNS vasculitis-like pattern. AJNR Am J Neuroradiol
12. Hung EC, Chim SS, Chan PK et al (2003) Detection of SARS coronavirus RNA in the cerebrospinal fluid of a patient with severe acute respiratory syndrome. Clin Chem 49(12):2108-2109

13. Katchanov J, Siebert E, Klingebiel R, Endres M (2010) Infectious vasculopathy of intracranial large- and medium-sized vessels in neurological intensive care unit: a clinico-radiological study. Neurocrit Care 12(3):369-374

14. Morassi M, Bagatto D, Cobelli M et al (2020) Stroke in patients with SARS-CoV-2 infection: case series. J Neurol:1-8

15. Muhammad S, Haasbach E, Kotchourko $M$ et al (2011) Influenza virus infection aggravates stroke outcome. Stroke. 42(3):783-791

16. Muhammad S, Petridis A, Cornelius JF, Hänggi D (2020) Letter to editor: severe brain haemorrhage and concomitant COVID-19 infection: a neurovascular complication of COVID-19. Brain Behav Immun S0889-1591(20):30802-30803

17. Nomura M, Mori K, Tamase A et al (2017) Pseudoaneurysm formation due to rupture of intracranial aneurysms: case series and literature review. Neuroradiol J 30(2):129-137. https://doi.org/10. $1177 / 1971400916684667$

18. Qin C, Zhou L, Hu Z et al (2020) Dysregulation of immune response in patients with COVID-19 in Wuhan, China. Clin Infect Dis:ciaa248

19. Rustemi O, Raneri F, Iannucci G, Volpin L, Segna A (2020, 2020) Aneurysmal subarachnoid hemorrhage in a SARS-CoV-2 positive testing: casual or causal? Br J Neurosurg:1-2

20. Sweid A, Hammoud B, Bekelis K et al (2020) Cerebral ischemic and hemorrhagic complications of coronavirus disease 2019. Int J Stroke 2020:1747493020937189

21. Umapathi T, Kor AC, Venketasubramanian N et al (2004) Large artery ischaemic stroke in severe acute respiratory syndrome (SARS). J Neurol 251(10):1227-1231

22. Wilkinson IM (1972) The vertebral artery. Extracranial and intracranial structure. Arch Neurol 27(5):392-396

23. World Health Organization (2019) Report of the WHO-China Joint Mission on Coronavirus Disease 2019 (COVID-19): https:/www. who.int/docs/default-source/coronaviruse/who-china-jointmission-on-covid-19-final-report.pdf

24. Wu YC, Chen CS, Chan YJ (2020) The outbreak of COVID-19: an overview. J Chin Med Assoc 83(3):217-220

25. Yonekawa Y, Zumofen D, Imhof HG, Roth P, Khan N (2008) Hemorrhagic cerebral dissecting aneurysms: surgical treatments and results. Acta Neurochir Suppl 103:61-69

26. Zulli A, Burrell LM, Buxton BF, Hare DL (2008) ACE2 and AT4R are present in diseased human blood vessels. Eur J Histochem 52(1):39-44

Publisher's note Springer Nature remains neutral with regard to jurisdictional claims in published maps and institutional affiliations. 